\title{
Treatment of Mild Hyperkalemia in Hospitalized Patients: An Unnecessary Practice?
}

\author{
Tracy A Freeze, Leanne Skerry, Emily Kervin, Rosemary Nunn, Jennifer Woodland, Natasha Hanson, \\ and Martin MacKinnon
}

Can J Hosp Pharm. 2021;74(3):269-76

DOI: 10.4212/cjhp.v74i3.3154

\begin{abstract}
Background: Sodium polystyrene sulfonate (SPS) is one of the most commonly used treatments for mild hyperkalemia. Other treatments include insulin, sodium bicarbonate, and salbutamol, which may be given alone or in combination. The results of research examining treatment effectiveness for mild hyperkalemia (e.g., the ability of SPS to achieve normokalemia) thus far have been inconsistent. Given that the effectiveness of treatment for mild hyperkalemia is debatable, new research is needed.
\end{abstract}

Objective: To determine whether treatment of hospitalized patients with mild hyperkalemia (using SPS or another approach, relative to no treatment) was associated with achievement of normokalemia (serum potassium $<5.1 \mathrm{mmol} / \mathrm{L}$ ).

Methods: For this retrospective, quasi-experimental study, hospitalized patients with index serum potassium level between 5.1 and $6.4 \mathrm{mmol} / \mathrm{L}$ were identified. Post-index serum potassium level within 24 hours was dichotomized ( $<5.1$ or $\geq 5.1 \mathrm{mmol} / \mathrm{L}$ ). Pre-index serum creatinine and serum potassium levels were recorded as the average of the first 5 values immediately before the index potassium value. For each patient, treatment was categorized as no treatment, SPS treatment, or other treatment strategy.

Results: Among the 1944 patients included in the analysis, the average age was 66.8 (standard deviation 13.5) years; 605 (31.1\%) of the patients were women and 1339 (68.9\%) were men. Logistic regression results indicated that patients who were female and/or had higher preindex serum potassium were less likely to return to normokalemia within 24 hours after the time of the index serum potassium value. Treatment category was not a statistically significant predictor of the achievement of normokalemia. Most patients with mild hyperkalemia ( $>74.5 \%$ in each treatment category) achieved normokalemia, whether or not they received treatment.

Conclusions: The findings of this study suggest that although followup is required for mild hyperkalemia in hospitalized patients, active treatment may be unnecessary.

Keywords: creatinine, hyperkalemia, potassium, treatment outcome, polystyrenes

\section{RÉSUMÉ}

Contexte : Le sulfonate de polystyrène de sodium (SPS) est I'un des traitements les plus communément utilisés pour l'hyperkaliémie légère. D'autres traitements comprennent l'insuline, le bicarbonate de sodium et le salbutamol, qui peuvent être administrés seuls ou ensemble. Les résultats des recherches se penchant sur l'efficacité des traitements de l'hyperkaliémie légère ( $p$. ex., la capacité du SPS à rétablir la normokaliémie) sont contradictoires jusqu'à présent. Étant donné que l'efficacité du traitement de l'hyperkaliémie légère est discutable, de nouvelles recherches sont nécessaires.

Objectif : Déterminer si le traitement des patients hospitalisés, présentant une hyperkaliémie légère, (à l'aide de SPS ou d'une autre approche, comparativement à l'absence de traitement) était associé à l'atteinte de la normokaliémie (potassium sérique $<5,1 \mathrm{mmol} / \mathrm{L}$ ).

Méthodes : Des patients hospitalisés, dont l'indice de concentration sérique de potassium se situait entre 5,1 et 6,4 $\mathrm{mmol} / \mathrm{L}$, ont été identifiés pour participer à cette étude rétrospective quasi expérimentale. La concentration sérique de potassium mesurée dans les 24 heures après le diagnostic d'hyperkaliémie légère a été dichotomisée $(<5,1$ ou $\geq 5,1 \mathrm{mmol} / \mathrm{L}$ ). Les indices de concentrations sériques de créatinine et de potassium avant le diagnostic d'hyperkaliémie légère ont été obtenus par la moyenne des cinq premières valeurs situées immédiatement avant celle de la concentration de potassium. Le classement du traitement de chaque patient était le suivant : Aucun traitement, Traitement par SPS ou Autre stratégie de traitement.

Résultats : L'âge moyen des 1944 patients inclus dans l'analyse était de 66,8 ans (écart type 13,5); 605 (31,1\%) d'entre eux étaient des femmes et $1339(68,9 \%)$ des hommes. Les résultats de la régression logistique indiquaient que les patientes, donc les femmes, qui avaient un indice sérique de potassium plus élevé au moment du diagnostic, avaient moins de chances de retourner à la normokaliémie dans les 24 heures après l'instant de la mesure de la valeur de l'indice sérique de potassium. La catégorie de traitement n'était pas une variable prédictive statistiquement significative de l'atteinte de la normokaliémie. La plupart des patients présentant une hyperkaliémie légère (> 74,5\% dans chaque catégorie de traitement) atteignaient la normokaliémie, qu'ils aient reçu ou non un traitement.

Conclusions : Les résultats de cette étude laissent entendre que, malgré la nécessité d'un suivi des patients hospitalisés en cas d'hyperkaliémie légère, un traitement actif pourrait s'avérer inutile.

Mots-clés : créatinine, hyperkaliémie, potassium, résultat du traitement, polystyrènes 


\section{INTRODUCTION}

Hyperkalemia, or levels of serum potassium above the normal range, ${ }^{1,2}$ is a potentially serious condition. ${ }^{3-6}$ According to one Canadian study, hyperkalemia was associated with $2.6 \%$ of emergency department visits and $3.5 \%$ of hospital admissions. ${ }^{7}$ One of the most harmful consequences of hyperkalemia is its potential impact on cardiac activity (e.g., serious acute cardiac arrhythmias, conduction abnormalities). ${ }^{8,9}$ Thus, severe hyperkalemia is considered a medical emergency that requires urgent treatment. ${ }^{8,9}$ For patients with severe hyperkalemia, clinicians administer medication to reduce potassium levels as quickly as possible. ${ }^{10}$ For patients with mild hyperkalemia, clinicians may administer medication, or they may wait to see if the patient's serum potassium returns to normal levels without pharmaceutical intervention. ${ }^{11}$ Although previous research supports intervention for severe hyperkalemia (i.e., in cases of medical emergency), ${ }^{12}$ there is a lack of research about the treatment of mild hyperkalemia. ${ }^{4,13}$

Pharmaceutical treatments for hyperkalemia include insulin, sodium bicarbonate, and salbutamol, administered alone or in combination. ${ }^{14,15}$, However, the most commonly used pharmaceutical intervention for the treatment of hyperkalemia is administration of the cation exchange resin sodium polystyrene sulfonate (SPS). ${ }^{16}$ This agent works by removing excess potassium from the body. Following administration, SPS takes effect within approximately 2 hours. ${ }^{17-19}$ The pharmacological effects last about 4 to 6 hours, although the duration of action varies with factors such as gastrointestinal (GI) transit time.,17,20 Given the delay in pharmaceutical effect, SPS is not used independently to manage hyperkalemia in cases where the patient is having a medical emergency that requires immediate treatment. ${ }^{12}$

Treatment with SPS carries some risk, ${ }^{9}$ as its use has been associated with adverse GI events in previous research. For instance, in a recent Canadian study of older adults, use of SPS was associated with a greater risk of hospital admission for severe GI issues within 30 days, relative to non-use. ${ }^{16}$ Similarly, Laureati and colleagues ${ }^{21}$ found that SPS initiation, without concomitant sorbitol, was related to a higher incidence of severe GI events among patients with advanced chronic kidney disease (CKD).

Despite common use of SPS for reducing serum potassium levels and its known potential risks, the effectiveness of this agent, especially for mild hyperkalemia, has been questioned. ${ }^{6,9,20}$ For instance, in a randomized controlled trial of 33 patients with CKD and mild hyperkalemia, treatment with $30 \mathrm{~g}$ of SPS by oral administration for 7 days was effective in reducing potassium levels but was not more effective than placebo in achieving normokalemia. ${ }^{22}$ Therefore, it is also important to consider and weigh the evidence concerning this drug's safety in addition to its small therapeutic effects. $^{2}$
Previous research on the treatment of hyperkalemia has focused on non-mild hyperkalemia and patients with CKD. Few studies, if any, have examined treatment in a broader range of patients or the achievement of normokalemia in patients with mild hyperkalemia. The primary purpose of this study was to determine whether treatment, primarily SPS, was associated with achievement of normokalemia (serum potassium $<5.1 \mathrm{mmol} / \mathrm{L}$ ) in hospitalized patients with mild hyperkalemia. Given that Lepage and others ${ }^{22}$ found no statistically significant difference in the achievement of normokalemia between groups receiving either a placebo or SPS, we hypothesized that there would be no statistically significant differences among groups receiving either no treatment, SPS alone, or other treatment (e.g., salbutamol, salbutamol and SPS combined) in a sample of patients with mild hyperkalemia.

\section{METHODS}

\section{Data Source and Procedure}

Approval for this retrospective quasi-experimental cohort study was obtained, before study initiation, through the Research Ethics Board (REB) of Horizon Health Network. A waiver of informed consent for secondary use of data was approved by the REB. The study site was a tertiary care hospital in Saint John, New Brunswick, with 524 inpatient beds. Data were retrieved from electronic hospital records for patients older than 19 years of age who were admitted to hospital between November 2009 and December 2018 and who had at least 1 serum potassium level of $5.1 \mathrm{mmol} / \mathrm{L}$ or above. The retrospective study period was selected on the basis of availability of electronic patient data; such data were unavailable for patients admitted before November 2009. For patients who were admitted more than once during the study period, only the first admission was included.

For this study, normokalemia was defined as serum potassium levels between 3.5 and $5.09 \mathrm{mmol} / \mathrm{L}$, and mild hyperkalemia as levels between 5.1 and $6.4 \mathrm{mmol} / \mathrm{L}$. These categories follow the work of Fordjour and others. ${ }^{23}$ Patients with pseudohyperkalemia (as identified by a laboratory hemolysis tag), end-stage renal disease (e.g., patients with $\mathrm{CKD}$, needing dialysis or kidney transplant to survive), acidosis (arterial or venous blood $\mathrm{pH}<7.2$ ), severe hyperkalemia (potassium serum level $>6.4 \mathrm{mmol} / \mathrm{L}$ ), or missing data (e.g., pre-index serum creatinine [SCr]; see below for the definition of "index") and those undergoing hemodialysis were excluded from the analyses.

\section{Study Variables}

The covariates collected for the current study included age, sex, pre-index serum potassium level, and pre- and postindex SCr levels. The index serum potassium level was defined as the first valid serum potassium value of $5.1 \mathrm{mmol} / \mathrm{L}$ 
or above during a patient's first hospital admission. The pre-index serum potassium and pre-index $\mathrm{SCr}$ levels were defined as the averages of the 5 serum potassium values and the $5 \mathrm{SCr}$ values, respectively, immediately preceding the index potassium value. The post-index SCr value was the average of all $\mathrm{SCr}$ values measured in the 24 -hour period after the index serum potassium level. The pre-index values were collected because of their potential clinical relevance and possible relation to treatment outcomes. They were included as covariates to statistically control for potential confounders related to differences in illness severity.

The independent variable was treatment for mild hyperkalemia. The 3 groups were no treatment, SPS treatment, and other treatment. The "other treatment" category consisted of treatments other than SPS alone; treatments in this category could include SPS in combination with other treatments for mild hyperkalemia (e.g., SPS, insulin, and salbutamol within the 24-hour follow-up period). Patients who were also receiving concurrent treatment for diseases such as diabetes and asthma were included in the analyses. For example, a patient who was receiving insulin on a regular basis for diabetes but did not receive any treatment for mild hyperkalemia was included in the "no treatment" category.

The dependent variable was the achievement of normokalemia, determined by the post-index serum potassium value. If the final serum potassium value was less than $5.1 \mathrm{mmol} / \mathrm{L}$, normokalemia was deemed to have been achieved. If the post-index serum potassium was $5.1 \mathrm{mmol} / \mathrm{L}$ or above, normokalemia was deemed not to have been achieved.

\section{Data Preparation}

Before the analysis, the data were examined for accuracy using descriptive statistics. ${ }^{24}$ Although there were outliers in the serum potassium and SCr levels, the data were determined to be accurate. Bivariate scatter plots and Q-Q plots were examined for normality, linearity, and homoscedasticity. Pre- and post-index SCr values displayed evidence of non-normality, nonlinearity, and heteroscedasticity. Given the large number of outliers and the accuracy of these data, a $\log$ transformation was performed. Subsequent testing indicated that the log-transformed pre- and post-index SCr values were relatively normally distributed and linear, although some minor heteroscedasticity remained. There were no missing values, as any case that did not include essential values was excluded, as per the research protocol. Multivariate outliers were identified using Mahalanobis distance measurements.

\section{Data Analysis}

A binary logistic regression analysis was conducted, with serum potassium within 24 hours after the index value (categorized as $\geq 5.1$ or $<5.1 \mathrm{mmol} / \mathrm{L}$ ) as the dependent variable and treatment category (i.e., no treatment, SPS treatment, other treatment) as the independent variable.
All assumptions of binary logistic regression analysis were examined, including multicollinearity, adequacy of expected frequencies, and ratio of cases to variables. Covariates were entered stepwise before testing of the independent variable. The covariates were pre-index serum potassium, transformed pre-index SCr, age, and sex. Post-index SCr was not included due to issues with multicollinearity, as described below. Linearity in the logit was tested according to the procedures outlined by Tabachnick and Fidell, ${ }^{24}$ and no serious violations were observed. An analysis of residuals indicated that there were no outliers in the solution.

\section{RESULTS}

\section{Descriptive Analyses}

Of the initial sample of 11014 patients, only 1997 met the study criteria (see Figure 1 for flow chart). Fifty-three patients were identified as multivariate outliers, and these were excluded from the final analysis. These multivariate outliers differed from the rest of the sample in terms of having extreme values for both pre- and post-index SCr. As a result, after removal of the outliers, the maximum preindex SCr declined from 1528 to $506 \mu \mathrm{mol} / \mathrm{L}$ and the maximum post-index SCr declined from 1332 to $731 \mu \mathrm{mol} / \mathrm{L}$.

The final sample of 1944 patients consisted of 605 women (31.1\%) and 1339 men (68.9\%), with a mean age of 66.8 (standard deviation [SD] 13.5) years. Table 1 outlines patients' demographic characteristics and median serum potassium levels by sex and treatment group, along with other outcomes. Only $22.8 \%$ of the patients received treatment $\left(n_{\mathrm{SPS}}=203 ; n_{\mathrm{Other}}=240\right)$ (Table 1$)$. The most common SPS dose was $30 \mathrm{~g}(n=126)$, and the most common method of administration was oral $(n=163)$. Other doses included $15 \mathrm{~g}(n=21), 20 \mathrm{~g}(n=4), 25 \mathrm{~g}(n=1), 40 \mathrm{~g}(n=2)$, and $60 \mathrm{~g}$ $(n=39)$, and other administration methods included rectal $(n=35)$ and enteral $(n=4)$. Insulin was the most commonly administered treatment in the "other treatment" category $(80.4 \%, n=193)$. The remaining treatments in the "other treatment" category were salbutamol and sodium bicarbonate alone or in combination with either insulin and/or SPS $(19.6 \%, n=47)$.

For all 3 groups, the median serum potassium levels decreased from index to post-index measurement, and most patients achieved normokalemia, regardless of treatment category (1171 [78.0\%] of 1501 in the "no treatment" group; 160 [78.8\%] of 203 in the SPS treatment group; 179 [74.6\%] of 240 in the "other treatment" group).

The median time from initial pre-index $\mathrm{SCr}$ value to index potassium value was 2.89 (interquartile range [IQR] 1.20-6.22) days, and the median time from initial pre-index to index potassium value was 2.73 (IQR 1.14-6.08) days. For the patients who received SPS or other treatment, the average time from treatment to post-index serum potassium value was 13.85 (SD 5.81) hours. 


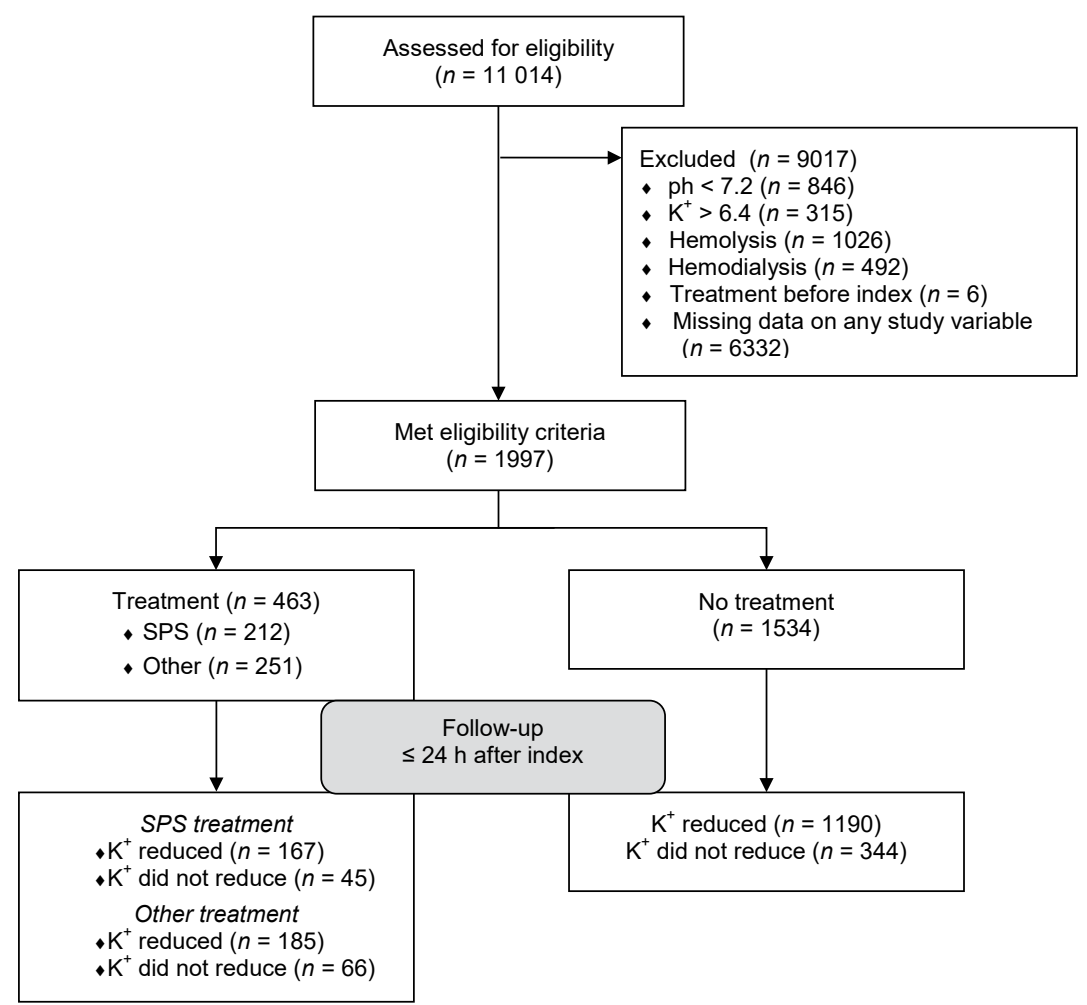

FIGURE 1. Participant flow diagram for study cohort before data analysis. SPS = sodium polystyrene sulfonate.

TABLE 1. Characteristics of 1944 Participants Included in the Final Analysis, by Treatment for Mild Hyperkalemia and Sex

Treatment Group; Median (IQR)

\begin{tabular}{|c|c|c|c|c|c|c|c|}
\hline \multirow[b]{2}{*}{ Characteristic } & \multicolumn{2}{|c|}{ No Treatment $(n=1501)$} & \multicolumn{2}{|c|}{ SPS $(n=203)$} & \multicolumn{2}{|c|}{ Other $(n=240)$} & \multirow{2}{*}{$\begin{array}{c}\text { Total } \\
(n=1944)\end{array}$} \\
\hline & Women & Men & Women & Men & Women & Men & \\
\hline \multicolumn{8}{|l|}{ Demographic } \\
\hline Age (years) $($ mean $\pm S D)$ & $69.6 \pm 14.7$ & $65.3 \pm 13.2$ & $71.9 \pm 13.1$ & $67.4 \pm 10.9$ & $65.8 \pm 14.5$ & $65.1 \pm 11.6$ & $66.8 \pm 13.5$ \\
\hline Weight $^{b}(\mathrm{~kg})$ & $\begin{array}{c}69.8 \\
(57.6-82.3)\end{array}$ & $\begin{array}{c}84.5 \\
(73.6-97.6)\end{array}$ & $\begin{array}{c}77.4 \\
(58.0-95.2)\end{array}$ & $\begin{array}{c}87.7 \\
(78.7-102.7)\end{array}$ & $\begin{array}{c}76.3 \\
(62.9-86.5)\end{array}$ & $\begin{array}{c}89.3 \\
(78.6-102.5)\end{array}$ & $\begin{array}{c}84.3 \\
(70.0-96.0)\end{array}$ \\
\hline Height $^{c}(\mathrm{~m})$ & $1.6(1.5-1.7)$ & $1.7(1.7-1.8)$ & $1.6(1.5-1.7)$ & $1.7(1.7-1.8)$ & $1.6(1.5-1.6)$ & $1.7(1.7-1.8)$ & $1.7(1.6-1.8)$ \\
\hline \multicolumn{8}{|l|}{ Potassium (mmol/L) } \\
\hline Pre-index & $4.4(4.1-4.6)$ & $4.4(4.2-4.6)$ & $4.5(4.3-4.7)$ & $4.5(4.3-4.6)$ & $4.4(4.2-4.6)$ & $4.5(4.3-4.6)$ & $4.4(4.2-4.6)$ \\
\hline Index & $5.2(5.1-5.4)$ & $5.2(5.1-5.4)$ & $5.5(5.3-5.8)$ & $5.4(5.2-5.6)$ & $5.4(5.2-5.7)$ & $5.4(5.3-5.8)$ & $5.3(5.1-5.5)$ \\
\hline Post-index & $4.7(4.4-5.1)$ & $4.7(4.4-5.0)$ & $4.8(4.3-5.1)$ & $4.6(4.2-4.9)$ & $4.7(4.3-5.0)$ & $4.8(4.6-5.1)$ & $4.7(4.4-5.0)$ \\
\hline \multicolumn{8}{|l|}{ Creatinine $(\mu \mathrm{mol} / \mathrm{L})$} \\
\hline Pre-index & $\begin{array}{c}82.0 \\
(60.5-117.0)\end{array}$ & $\begin{array}{c}88.6 \\
(73.5-114.9)\end{array}$ & $\begin{array}{c}107.7 \\
(77.8-146.5)\end{array}$ & $\begin{array}{c}98.7 \\
(80.5-133.6)\end{array}$ & $\begin{array}{c}88.0 \\
(64.05-128.8)\end{array}$ & $\begin{array}{c}98.0 \\
(82.0-124.0)\end{array}$ & $\begin{array}{c}90.0 \\
(71.8-118.0)\end{array}$ \\
\hline Post-index & $\begin{array}{c}85.0 \\
(57.5-127.0)\end{array}$ & $\begin{array}{c}91.0 \\
(73.0-122.7)\end{array}$ & $\begin{array}{c}122.0 \\
(81.5-187.7)\end{array}$ & $\begin{array}{c}123.0 \\
(85.0-180.0)\end{array}$ & $\begin{array}{c}120.0 \\
(74.0-173.0)\end{array}$ & $\begin{array}{c}120.5 \\
(93.0-162.0)\end{array}$ & $\begin{array}{c}95.7 \\
(72.0-135.0)\end{array}$ \\
\hline \multicolumn{8}{|c|}{ Post-index potassium (no. of participants) } \\
\hline$\geq 5.1 \mathrm{mmol} / \mathrm{L}$ & 126 & 204 & 21 & 22 & 7 & 54 & 434 \\
\hline$<5.1 \mathrm{mmol} / \mathrm{L}$ & 371 & 800 & 42 & 118 & 38 & 141 & 1510 \\
\hline Total $n$ & 497 & 1004 & 63 & 140 & 45 & 195 & 1944 \\
\hline
\end{tabular}

$\mathrm{IQR}=$ interquartile range, $\mathrm{SD}=$ standard deviation, $\mathrm{SPS}=$ sodium polystyrene sulfonate.

aExcept where indicated otherwise.

bData for weight were missing for 312 participants.

'Data for height were missing for 518 participants. 


\section{Statistical Analyses}

A correlational analysis indicated potential issues with collinearity. More specifically, pre- and post-index SCr values were highly correlated $(r=0.81, p<0.001$ for the nontransformed scores; $r=0.83, p<0.001$ for the transformed scores). Therefore, transformed post-index SCr was not included in the statistical analysis. A test of the stepwise model of covariates against a constant-only model was statistically significant $\left(\chi^{2}=19.67, p<0.001\right)$ with transformed pre-index SCr entered first into the model, followed by sex and pre-index serum potassium value. Given that stepwise regression analyses remove variables unassociated with outcome, and given that age was unassociated with the outcome, age was removed from the analysis. The Nagelkerke $R^{2}$ was 0.015 (95\% confidence interval [CI] 0.005-0.026), ${ }^{25}$ indicating that approximately $1.5 \%$ of the variance in whether or not serum potassium levels declined within 24 hours was due to the 3 covariates of transformed pre-index SCr, sex, and pre-index serum potassium levels. Furthermore, the result of the Hosmer and Lemeshow test was nonsignificant $\left(\chi^{2}\right.$ $=7.57, p=0.48)$. Classification was acceptable, with $77.7 \%$ of the cases correctly classified. However, although $100 \%$ of the cases with reduced serum potassium levels were correctly classified, none of the cases with nonreduction in serum potassium levels were correctly classified. The addition of treatment did not improve the model $\left(\chi^{2}=2.03, p\right.$ $=0.36$ ). In other words, neither of the 2 active treatment groups predicted the achievement of normokalemia relative to no treatment.

Table 2 presents the regression coefficients, Wald statistics, and odds ratios (ORs) with 95\% CIs for each of the predictors. The pre-index serum potassium level significantly predicted the outcome: $\chi^{2}(1, n=1944)=3.88, p=0.049$. The OR for pre-index serum potassium level was 0.72 , indicating that the odds of a reduction in serum potassium to below $5.1 \mathrm{mmol} / \mathrm{L}$ within 24 hours was decreased with higher pre-index serum potassium levels. Sex significantly predicted outcome: $\chi^{2}(1, n=1944)=6.76, p=0.009$. The OR for male sex was 1.35 . Therefore, being male was associated with a 1.35 times greater odds of reduction of serum potassium to below $5.1 \mathrm{mmol} / \mathrm{L}$ within 24 hours. Finally, the transformed value for pre-index SCr significantly predicted outcome: $\chi^{2}(1, n=1944)=7.66, p=0.006$. The OR was 0.43 , indicating that the odds of a reduction in serum potassium to below $5.1 \mathrm{mmol} / \mathrm{L}$ within 24 hours were decreased with higher levels of the transformed pre-index SCr.

Follow-up independent-sample $t$ tests indicated a statistically significant difference in index serum potassium levels between each of the 2 treatment groups and the "no treatment" group $\left(t_{\mathrm{SPS}}=-8.93, p<0.001 ; t_{\mathrm{Other}}=-7.25, p<\right.$ 0.001). However, logistic regression examining the ability of treatment to predict outcome after controlling for index serum potassium level was nonsignificant: $\chi^{2}(2, n=1944)=$ $1.01, p=0.60$.

\section{DISCUSSION}

This study provides a detailed description of the treatment of mild hyperkalemia in a tertiary care hospital. The main finding was that neither SPS nor other treatment strategies predicted the achievement of potassium levels less than $5.1 \mathrm{mmol} / \mathrm{L}$ (normokalemia) relative to no treatment. Although neither treatment category had a statistically significant result, certain patient characteristics were found to be statistically significant predictors of the outcome, including pre-index serum potassium level, sex, and the transformed pre-index SCr level. Specifically, the odds of attaining normokalemia within 24 hours declined with higher pre-index serum potassium levels, higher levels of the transformed pre-index SCr, and female sex. Additionally, fewer patients than expected were treated for mild hyperkalemia. Of the 1944 patients included in the analysis, only $22.8 \%$ received either SPS or another form of treatment. Whether they received treatment or not, $74.6 \%$

TABLE 2. Stepwise Sequential Binary Logistic Regression Analysis of Potassium ( $\left.\mathrm{K}^{+}\right)$Reduction, as a Function of Patient Characteristics and Treatment

\begin{tabular}{|c|c|c|c|c|c|c|}
\hline Variable & $B$ & SE & Wald $\chi^{2}$ & \multicolumn{2}{|c|}{ Odds Ratio $(95 \% \mathrm{Cl})$} & $p$ Value \\
\hline \multicolumn{7}{|l|}{ Block 1} \\
\hline Pre-index $\mathrm{K}^{+}$ & -0.32 & 0.16 & 3.88 & 0.72 & $(0.52-0.97)$ & 0.049 \\
\hline Pre-index $\mathrm{SCr}^{\mathrm{a}}$ & -0.83 & 0.30 & 7.66 & 0.43 & $(0.24-0.78)$ & 0.006 \\
\hline Male sex & 0.30 & 0.12 & 6.76 & 1.35 & $(1.08-1.70)$ & 0.009 \\
\hline \multicolumn{7}{|l|}{ Block 2} \\
\hline SPS treatment ${ }^{b}$ & 0.13 & 0.18 & 0.52 & 1.14 & $(0.79-1.64)$ & 0.47 \\
\hline Other treatment ${ }^{c}$ & -0.18 & 0.16 & 1.25 & 0.83 & $(0.61-1.15)$ & 0.26 \\
\hline
\end{tabular}

$\mathrm{Cl}=$ confidence interval, $\mathrm{SCr}=$ serum creatinine, $\mathrm{SE}=$ standard error, $\mathrm{SPS}=$ sodium polystyrene sulfonate.

aPre-index $\mathrm{SCr}=$ transformed pre-index $\mathrm{SCr}$.

bSPS compared with no treatment.

Treatment other than SPS alone compared with no treatment. 
to $78.8 \%$ of hospitalized patients with mild hyperkalemia achieved normokalemia within 24 hours.

Our main finding of no significant difference between treatment and no treatment in the achievement of normokalemia, even after controlling for pre-index serum potassium and SCr levels, does not support previous research, which has found SPS to be effective. ${ }^{6,20,26,27}$ However, the previous studies had limitations that our study addresses. For instance, most of the previous studies had small sample sizes and no comparison group (e.g., placebo or no treatment), whereas our study had a large sample size and direct comparison with a group that received no treatment. At the same time, our research supports and extends the work of Lepage and others, ${ }^{22}$ who conducted a clinical trial with placebo comparison and, although the sample size was underpowered $(n=33)$, found no statistically significant difference between placebo and SPS in the reduction of serum potassium in patients with CKD. Our study had a much larger sample consisting of a wide range of hospitalized patients with mild hyperkalemia. Our findings also support the conclusions of Batterink and others, ${ }^{28}$ who conducted a retrospective review of hospital records for 138 patients. Although their findings indicated a statistically significant difference between no treatment and treatment with SPS, they noted that the treatment effect was small and might not be clinically important.

Nevertheless, it could be argued that differences in index serum potassium levels might account for our finding that treatment was not associated with achievement of normokalemia. In other words, perhaps patients with higher index serum potassium levels were treated and patients with lower index serum potassium levels were not treated, leading to confounding of the results by index serum potassium level. However, follow-up analyses, as described in the Results section, indicated that group differences in index serum potassium did not explain why treatment was not associated with the achievement of normokalemia. Furthermore, by using pre-index serum potassium and $\mathrm{SCr}$ levels as statistical controls, this study eliminated variability between groups that might have been due to differences in illness severity.

The finding that women were at greater risk of not achieving normokalemia within 24 hours of the index serum potassium was surprising, given that previous research has found that male sex is a risk factor for hyperkalemia. ${ }^{12}$ Overall, in our study, approximately 1 in 5 men (20.9\%) and 1 in 4 women (25.4\%) did not experience a return to normokalemia. The sex-based rates were similar for the patients who received no treatment, but different for the patients who received SPS or other treatment. Among patients who received SPS, $33.3 \%$ of women and $15.7 \%$ of men did not achieve normokalemia. Among patients in the "other treatment" group, $15.6 \%$ of women and $27.7 \%$ of men did not achieve normokalemia. Women in our study differed from men in other respects as well. For instance, women were older than men (average age 69.6 and 65.5 years, respectively). The age difference was most prominent for the SPS treatment group (71.9 and 67.4 years, respectively). In addition, the median transformed pre-index SCr was higher for women in the SPS treatment group than the overall median transformed pre-index SCr for the entire study group. Therefore, the finding that women were at higher risk of not achieving normokalemia might have been due to the age or pre-index SCr of the women in our sample and not to sex-related differences. Nonetheless, other researchers have found some evidence that women are at higher risk of hyperkalemia. For instance, Turgutalp and others, ${ }^{29}$ in their sample of Turkish patients, found that women were at higher risk of community-acquired hyperkalemia.

\section{Strengths and Limitations}

Relative to previous studies of this topic, this study was strengthened by the large sample of hospitalized patients with mild hyperkalemia. The larger sample enhanced our ability to generalize beyond the study sample and reduced the likelihood of type II error. Generalization was also improved by not limiting our sample to select patient populations (e.g., patients with CKD). In addition to including broad patient populations, our research had other pragmatic design features, ${ }^{30}$ such as the real-world examination of treatment versus no treatment in the reduction of serum potassium to below $5.1 \mathrm{mmol} / \mathrm{L}$.

Despite the many strengths of this study, its retrospective nature necessitates a discussion of limitations. One limitation of this real-world retrospective study was the lack of control over when blood samples were taken. Any serum potassium level reported within the 24-hour period following the index value was included; therefore, the timing of blood samples varied considerably among study participants. For patients who were given treatment, the follow-up serum potassium level may have been measured before the treatment could have any effect. Furthermore, if follow-up serum potassium was not reported within 24 hours after the index, it was not included in our analysis. Although it is possible that serum potassium decreased beyond the 24-hour time limit, the UK guidelines suggest that 6 hours is adequate time for treatment to be effective, ${ }^{3}$ and the average time from treatment to follow-up in the current study was more than 13 hours.

Another limitation of this study was lack of control over the dose of SPS (or other treatments). Variation in dose across the study sample and the use of different doses for the same patient within the 24-hour period of interest were possible, but most patients in the SPS group received the typical dose of $30 \mathrm{~g}$ by oral administration, and most patients in the "other treatment" group received insulin alone. In addition, patients might have been receiving medications for other illnesses. For instance, many of the 
patients had other illnesses (e.g., diabetes) and could have been receiving medications (e.g., insulin) for these illnesses as well. Although this lack of control over other medications and illnesses is a limitation, it may also be considered a strength of the study, given that it allows for the real-word examination of treatment for mild hyperkalemia. ${ }^{31}$

This study did not examine the effectiveness of administration of fluids or any of the more recent pharmaceutical treatments for hyperkalemia. Research on patiromer sorbitex calcium and sodium zirconium cyclosilicate (also known as ZS-9) has indicated that these medications are effective in managing hyperkalemia. ${ }^{32}$ Future research could investigate the administration of fluids and newer pharmaceutical treatments in the management of mild hyperkalemia, although our evidence suggests that pharmaceutical intervention may be unnecessary for most patients with mild hyperkalemia, given that $78 \%$ of those with no intervention achieved normokalemia. Investigating unobserved group differences in the trajectory of serum potassium change could also be addressed in future studies. If unobserved groups (i.e., latent classes) are found, variables differentiating the groups could be assessed to help identify patients with mild hyperkalemia who are at risk of further exacerbation or non-normalization of serum potassium levels. Identifying these patients would have important clinical implications and could help identify those patients who are in need of intervention.

\section{CONCLUSION}

The findings of this study demonstrated that most patients were not treated for mild hyperkalemia in the 24-hour follow-up period after documentation of hyperkalemia, and there was no statistically significant association between treatment and achievement of normokalemia relative to no treatment, regardless of whether patients were treated with SPS or another hyperkalemia-reducing strategy. In a recent editorial, Parks and Grady remarked that SPS should not be used to reduce serum potassium levels. ${ }^{30}$ Our research supports that conclusion, at least for patients with mild hyperkalemia. For these patients, our research suggests that, although follow-up is required, elevated serum potassium levels may resolve within 24 hours without intervention. Thus, for most hospitalized patients, treatment of mild hyperkalemia may be unnecessary.

\section{References}

1. Sterns RH, Rojas M, Bernstein P, Chennupati S. Ion-exchange resins for the treatment of hyperkalemia: are they safe and effective? J Am Soc Nephrol. 2010;21(5):733-5.

2. Mueller ZT, Crannage AJ. Evaluation of appropriate sodium polystyrene sulfonate use with a hyperkalemia order set: a pilot study. J Pharm Pract. 2019;32(5):493-8.

3. Alfonzo A, Soar J, MacTier R, Fox J, Shillday I, Nolan J, et al. Treatment of acute hyperkalaemia in adults. U K Renal Association; 2014.
4. Yousaf F, Spinowitz B, Charytan C. Management of mild hyperkalemia with sodium polystyrene sulfonate: is it necessary? Clin Pract. 2014;11(5):501-7.

5. Ingelfinger JR. A new era for the treatment of hyperkalemia? $N$ Engl J Med. 2015;372(3):275-7.

6. Kessler C, Ng J, Valdez K, Xie H, Geiger B. The use of sodium polystyrene sulfonate in the inpatient management of hyperkalemia. $J$ Hosp Med. 2011;6(3):136-40.

7. Fleet JL, Shariff SZ, Gandhi S, Weir MA, Jain AK, Garg AX. Validity of the International Classification of Diseases 10th revision code for hyperkalaemia in elderly patients at presentation to an emergency department and at hospital admission. BMJ Open. 2012;2(6):e002011.

8. Putcha N, Allon M. Management of hyperkalemia in dialysis patients. Semin Dialysis. 2007;20(5):431-9.

9. Packham DK, Kosiborod M. Potential new agents for the management of hyperkalemia. Am J Cardiovasc Drugs. 2016;16(1):19-31.

10. Weisberg LS. Management of severe hyperkalemia. Crit Care Med. 2008;36(12):3246-51.

11. Chaitman M, Dixit D, Bridgeman MB. Potassium-binding agents for the clinical management of hyperkalemia. P T. 2016;41(1):43-50.

12. Montford JR, Linas S. How dangerous is hyperkalemia? J Am Soc Nephrol. 2017;28(11):3155-65.

13. Hagan AE, Farrington CA, Wall GC, Belz MM. Sodium polystyrene sulfonate for the treatment of acute hyperkalemia: a retrospective study. Clin Nephrol. 2016;85(1):38-43.

14. Sterns RH, Grieff M, Bernstein PL. Treatment of hyperkalemia: something old, something new. Kidney Int. 2016;89(3):546-54.

15. Dépret F, Peacock WF, Liu KD, Rafique Z, Rossignol P, Legrand M. Management of hyperkalemia in the acutely ill patient. Ann Intensive Care. 2019;9(1):32.

16. Noel JA, Bota SE, Petrcich W, Garg AX, Carrero JJ, Harel Z, et al. Risk of hospitalization for serious adverse gastrointestinal events associated with sodium polystyrene sulfonate use in patients of advanced age. JAMA Intern Med. 2019;179(8):1025-33.

17. Kim HJ, Han SW. Therapeutic approach to hyperkalemia. Nephron. 2002;92 Suppl 1:33-40.

18. Long B, Warix JR, Koyfman A. Controversies in management of hyperkalemia. J Emerg Med. 2018;55(2):192-205.

19. Pitt B, Bakris GL. New potassium binders for the treatment of hyperkalemia: current data and opportunities for the future. Hypertension. 2015;66(4):731-8.

20. Mistry M, Shea A, Giguere P, Nguyen ML. Evaluation of sodium polystyrene sulfonate dosing strategies in the inpatient management of hyperkalemia. Ann Pharmacother. 2016;50(6):455-62.

21. Laureati P, Xu Y, Trevisan M, Schalin L, Mariani I, Bellocco R, et al. Initiation of sodium polystyrene sulphonate and the risk of gastrointestinal adverse events in advanced chronic kidney disease: a nationwide study. Nephrol Dial Transplant. 2019;35(9):1518-26.

22. Lepage L, Dufour AC, Doiron J, Handfield K, Desforges K, Bell R, et al. Randomized clinical trial of sodium polystyrene sulfonate for the treatment of mild hyperkalemia in CKD. Clin J Am Soc Nephrol. 2015; 10(12):2136-42.

23. Fordjour KN, Walton T, Doran JJ. Management of hyperkalemia in hospitalized patients. Am J Med Sci. 2014;347(2):93-100.

24. Tabachnick BG, Fidell LS. Using multivariate statistics. Pearson Education; 2013.

25. Steiger JH, Fouladi RT. R2: a computer program for interval estimation, power calculations, sample size estimation, and hypothesis testing in multiple regression. Behav Res Methods Instrum Comput. 1992;24:581-2.

26. Georgianos PI, Liampas I, Kyriakou A, Vaios V, Raptis V, Savvidis N, et al. Evaluation of the tolerability and efficacy of sodium polystyrene sulfonate for long-term management of hyperkalemia in patients with chronic kidney disease. Int Urol Nephrol. 2017;49(12):2217-21.

27. Hunt TV, DeMott JM, Ackerbauer KA, Whittier WL, Peksa GD. Single-dose sodium polystyrene sulfonate for hyperkalemia in chronic kidney disease or end-stage renal disease. Clin Kidney J. 2019;12(3): 408-13. 
28. Batterink J, Lin J, Au-Yeung SHM, Cessford T. Effectiveness of sodium polystyrene sulfonate for short-term treatment of hyperkalemia. Can J Hosp Pharm. 2015;68(4):296-303.

29. Turgutalp K, Bardak S, Helvacı I, İşgüzar G, Payas E, Demir S, et al. Community-acquired hyperkalemia in elderly patients: risk factors and clinical outcomes. Renal Failure. 2016;38(9):1405-12.

30. Parks M, Grady D. Sodium polystyrene sulfonate for hyperkalemia. JAMA Intern Med. 2019;179(8):1023-4.

31. Ford I, Norrie J. Pragmatic trials. N Engl J Med. 2016;375(5):454-63.

32. Meaney CJ, Beccari MV, Yang Y, Zhao J. Systematic review and meta-analysis of patiromer and sodium zirconium cyclosilicate: a new armamentarium for the treatment of hyperkalemia. Pharmacotherapy. 2017;37(4):401-11.
Tracy A Freeze, PhD, is with Horizon Health Network, Saint John Regional Hospital, Saint John, New Brunswick.

Leanne Skerry, MA, is with Horizon Health Network, Saint John Regional Hospital, Saint John, New Brunswick.

Emily Kervin, MA, is with Horizon Health Network, Saint John Regional Hospital, Saint John, New Brunswick.

Rosemary Nunn, BN, RN, is with Saint John Regional Hospital, Saint John, New Brunswick.

Jennifer Woodland, PhD, is with Horizon Health Network, Saint John Regional Hospital, Saint John, New Brunswick.

Natasha Hanson, PhD, is with Horizon Health Network, Saint John Regional Hospital, Saint John, New Brunswick.

Martin MacKinnon, MD, FRCPC, is with the Department of Nephrology, Horizon Health Network, New Brunswick, and the Department of Medicine, Dalhousie University, Halifax, Nova Scotia.

Competing interests: None declared.

Address correspondence to:

Dr Martin MacKinnon

Department of Nephrology, Saint John Regional Hospital

Horizon Health Network

400 University Avenue

Saint John NB E2L 4L2

email: Martin.MacKinnon@horizonnb.ca

Funding: None received.

Acknowledgment: Nonmonetary support for this study was provided by Horizon Health Network's SOAR (Support Opportunities and Assistance for Research) Program.

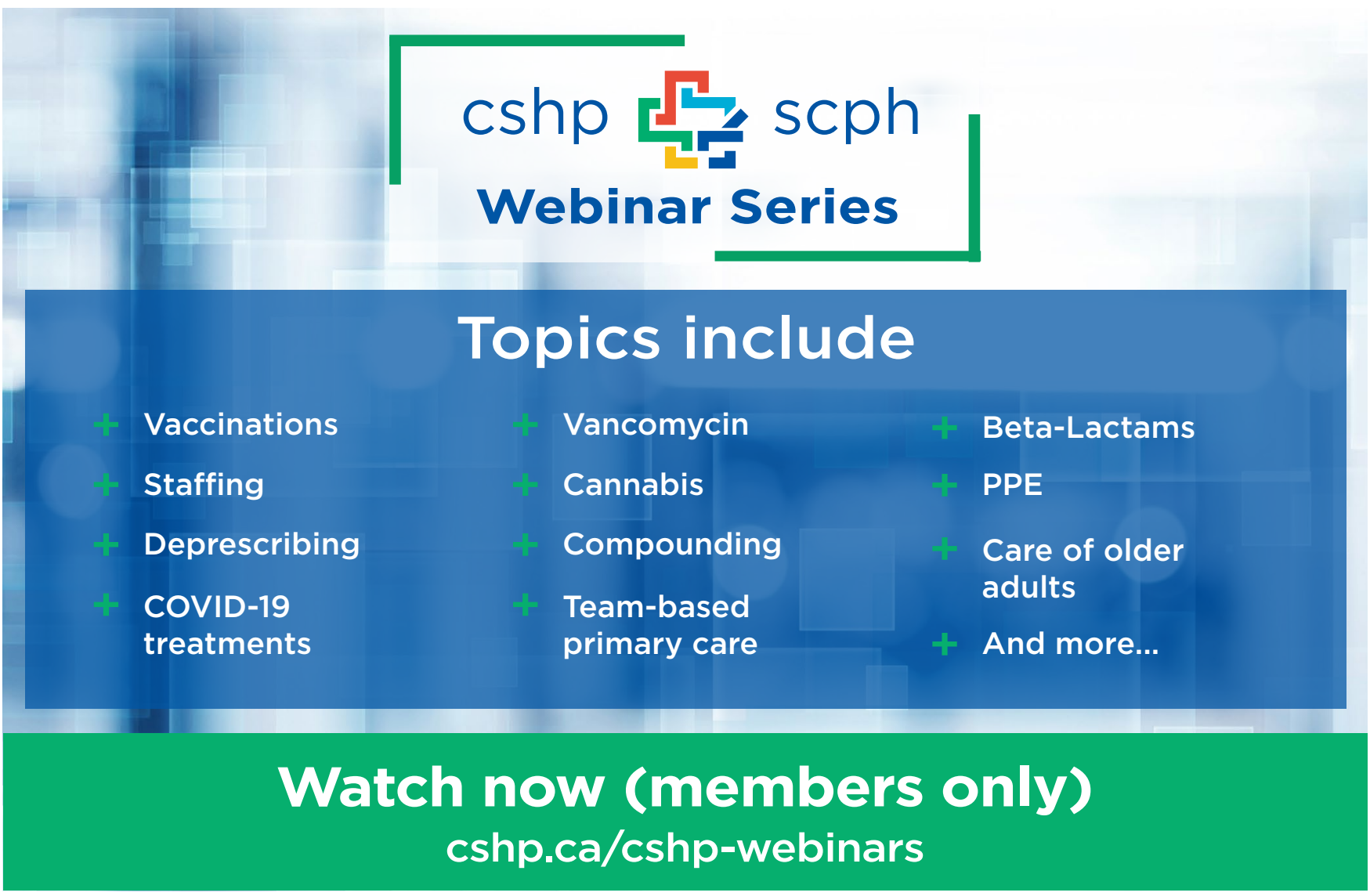

